\title{
Electron Emission for Electric Propulsion: Reducing Power by Mitigating Space Charge Limits
}

\author{
David P. Morris ${ }^{*}$ and Brian E. Gilchrist ${ }^{\dagger}$ \\ University of Michigan, Ann Arbor, MI, 48109
}

\begin{abstract}
Electron emission is a requirement for most forms of space electric propulsion, as well as many other space applications. For many such missions minimizing system power is critical to the viability of the mission. The generation of electrons requires an amount of power that varies depending on the source (cold cathode and thermionic sources are considered here, not hollow cathode or other sources requiring consumables) but regardless of the source, the electrons must leave as a beam that has sufficient power (velocity) to escape the spacecraft. The space charge limit refers to the maximum current that can cross a gap (the sheath between the spacecraft and the surrounding plasma for example) at a given velocity, and thus is a practical lower bound to the beam power required for a given current. For many systems, especially those using low-power electron sources, this means that power must be added to the electron beam, usually with biased grids. The minimization of this electron beam power requirement is the topic of this paper. Analytic solutions for the space charge limit in simple geometries have been developed up to three dimensions, and these demonstrate some basic performance tradeoffs. Less practical for the analytical approach are more complicated geometries and the addition of spacing and timing factors. These complications, however, can allow significant improvement in the space charge limit. Therefore, using particle-in-cell computer simulations (with the XOOPIC code developed at Berkley), a number of techniques for mitigating the space charge limit have been investigated, ranging from atypical geometries to spatial and chronological phasing of emission. Some of the advantages and disadvantages of some techniques, and the implications for electron emission system design, will be presented here.
\end{abstract}

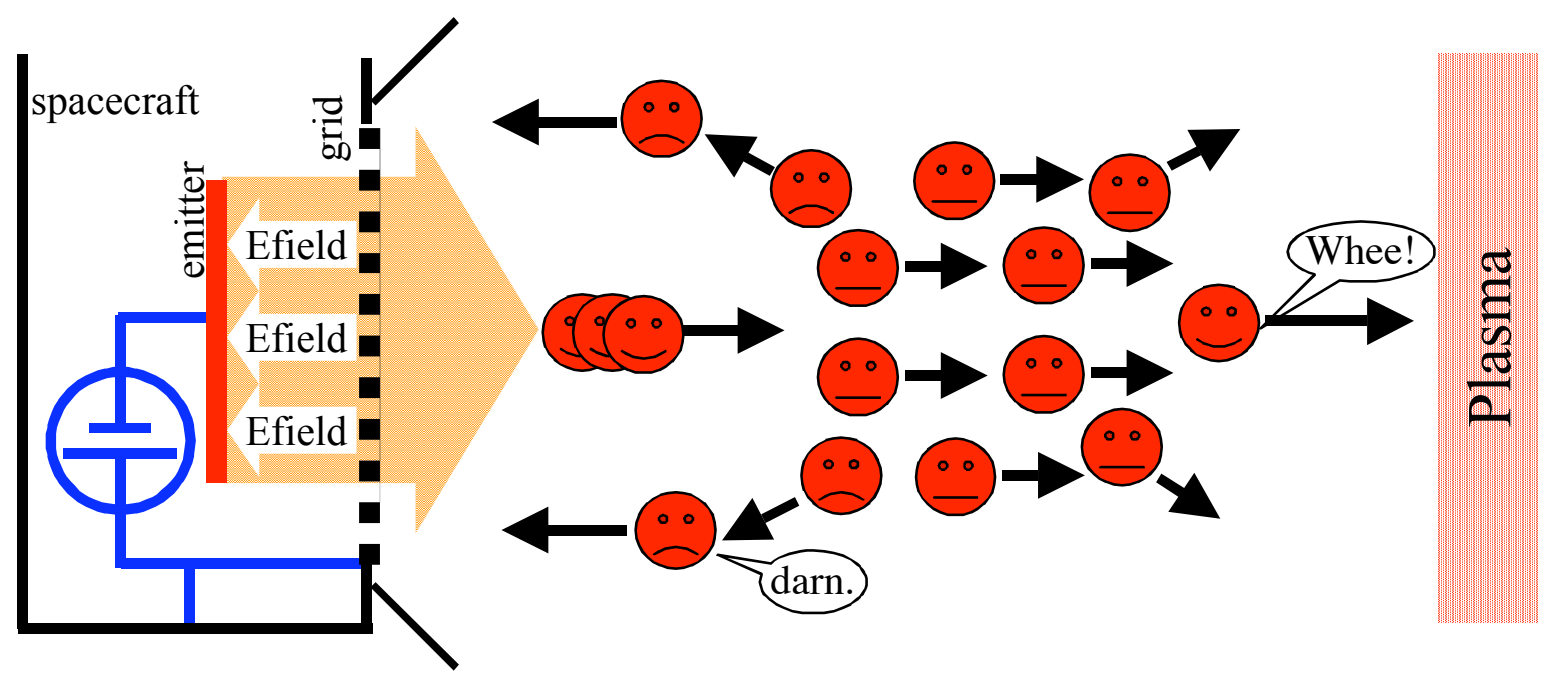

\footnotetext{
${ }^{*}$ PhD Student, Electrical Engineering and Space Systems, 2455 Hayward

${ }^{\dagger}$ Professor, Electrical Engineering and Space Systems, 2455 Hayward, AIAA Associate Fellow
} 


$\begin{array}{lll} & \text { Nomenclature } \\ \mathrm{FE} & =\text { field emitter } \\ \mathrm{EFES} & =\text { electron field emitter system } \\ \mathrm{SCL} & = & \text { space charge limit } \\ \mathrm{PIC} & = & \text { particle-in-cell } \\ \mathrm{Vgt} & = & \text { gate tip voltage } \\ \varepsilon_{0} & =\text { permittivity of free space } \\ \mathrm{e} & =\text { electron charge }[\mathrm{C}] \\ \mathrm{m}_{\mathrm{e}} & =\text { electron mass }[\mathrm{kg}] \\ \mathrm{T}_{\mathrm{o}} & =\text { electron emission energy }[\mathrm{eV}] \\ \mathrm{D} & =\text { gap spacing }[\mathrm{m}] \\ \mathrm{V} & =\text { gap voltage }[\mathrm{V}] \\ \mathrm{W} & =\text { emitter width } \\ \mathrm{r}_{\mathrm{b}} & =\text { emitter radius } \\ \mathrm{A} & = & \text { emitter area }\left[\mathrm{m}^{2}\right] \\ \mathrm{S} & = & \text { sheath size }[\mathrm{m}] \\ \mathrm{J}_{\mathrm{CL}}(\mathrm{N}) & = & \mathrm{N} \text { dimensional Child-Langmuir current limit }\left[\mathrm{A} / \mathrm{m}^{2}\right]\end{array}$

\section{Introduction}

The topic of this paper is improvement of the efficiency of electron emission, primarily applied to in-space applications, via analysis of the space charge limit and the development of techniques to mitigate, improve, or get around this limit. Any system emitting electrons at low energy (relative to the emitted current) potentially faces the space charge limit, a point at which the interactions of the emitted electrons pushing on each other becomes sufficient to slow down and reflect the beam. This limit is especially salient to small spacecraft in-space electric propulsion and other space applications where power spent on emission is at a premium. The primary motivation of this work is for the development of cold cathode or field effect electron emitters (FEs) because they are particularly capable of high current low power emission, but the results are applicable to thermionic and other types of emitters as well.

I will begin with an overview of the space charge limit and how it effects emission. I will briefly cover analytic solutions to the space charge limit developed from the early 1900s to today, ranging from 1 dimensional to 3 dimensional, and what these imply. I will then cover some of the techniques to improve upon these limits, including emitter segmentation and defocus rings. I will show the results of these derived primarily via PIC simulations in XOOPIC, which I will describe as well. I will also present some experimental work done in support of this research.

\section{The Space Charge Limit}

The first electrons in a beam moving at accelerated velocity from an emitter move freely to the anode, out into the vacuum, or even more ideally across the positively biased sheath between the spacecraft and surrounding plasma. Subsequent electrons, however, see the negative field surrounding each electron in the initial portion of the beam. Like charges repel and these electrons are decelerated somewhat. Subsequent electrons decelerate even more. Below the space charge limit, in the steady state, there will be a bunching of electrons that have decelerated thusly somewhere in the gap between the emitter and the anode. Electrons decelerate as they approach this potential minimum, and accelerate once they are past it. The space charge limit occurs when then bunching reaches sufficient density relative to the emission velocity and other parameters that electrons come to complete stop. Once this happens, the density at this central point, this "virtual cathode", increases rapidly and new electrons are reflected back to the source. Because the development of the virtual cathode accelerates once electrons are reflected, reaching the space charge limit is a catastrophic event from an emission point of view. The virtual cathode grows large and the majority of the charge is reflected back to the source. Much more charge is emitted at epsilon below the space charge limit than at epsilon above. Thus identifying and avoiding this space charge limit is critical. The following figure illustrates this effect. 


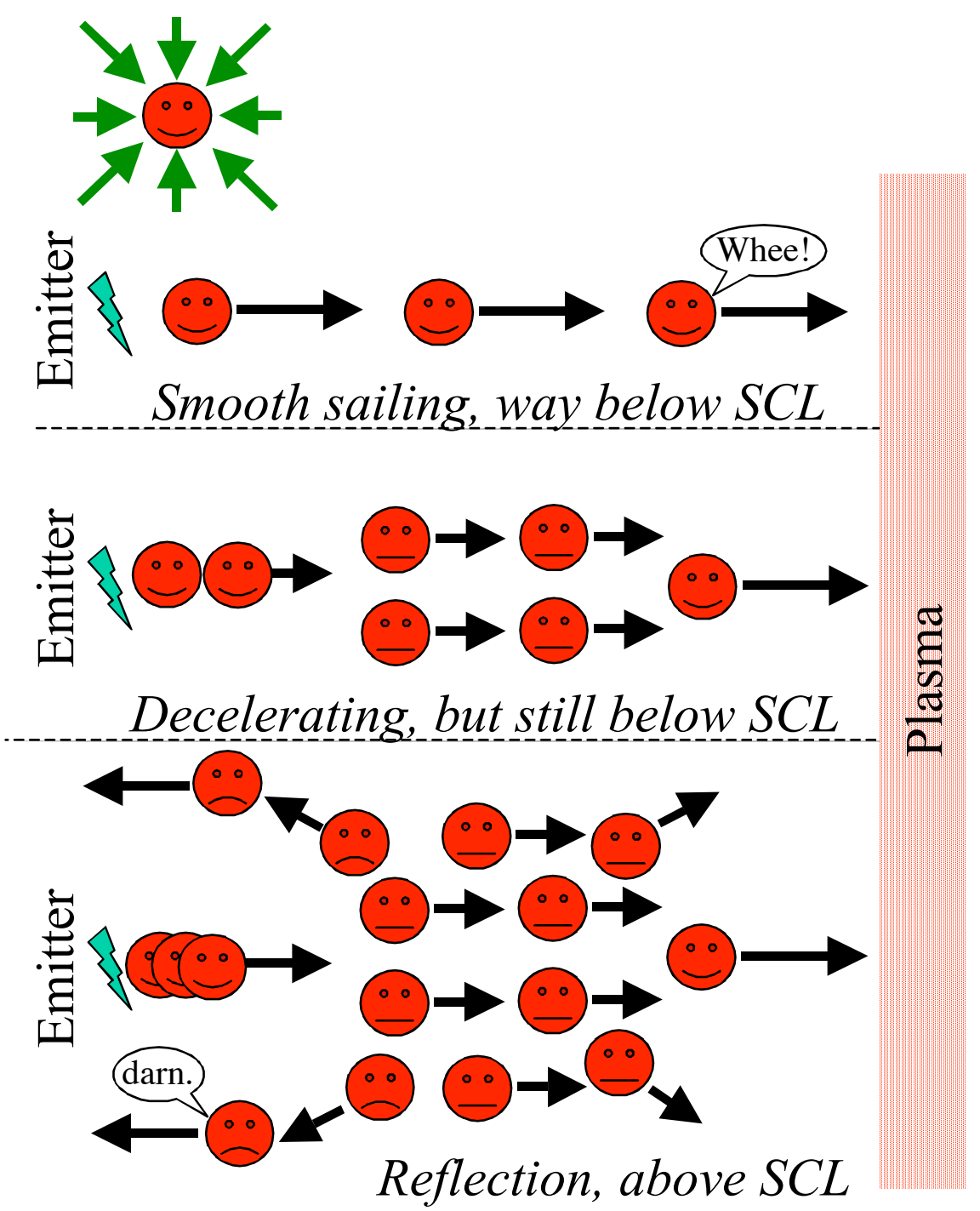

Figure 1. Graphical depiction of the space charge limit effect for electron emission from a spacecraft cathode into a surrounding plasma/anode for various beam densities- below, near, and above the space charge limit.

\section{Dimensional Space Charge Limit}

The simplest geometry for the space charge limit analysis is an infinite plane. This geometry was first studied in the early 1900s by Clement Dexter Child [1911], and by Irving Langmuir [1913]. Their research was inspired by electron emission in vacuum tubes, but the applications are far reaching. Using the continuity equation (aka Kirchoff's current law) that the current must be equal at every point between the plates, the force equation for charged particles in an electric field, Poisson's equation, $\mathrm{F}=\mathrm{ma}$, and calculus, they derived the following (converted to MKS units):

$$
J_{1 D}=\frac{4 \varepsilon_{0}}{9 D^{2}}\left(\frac{2 e}{m}\right)^{1 / 2} V^{3 / 2}
$$

$\left[\mathrm{A} / \mathrm{m}^{\wedge} 2\right]$

The basic one dimensional space charge limit equation has been studied and expanded upon a great deal in the intervening years. In one dimension with an initial emission velocity assumed, the space-charge current limited condition is given as follows [Luginsland, 1998]: 


$$
I_{C L}(1)=\frac{4 \varepsilon_{o}}{9 e} \sqrt{\frac{2}{m_{e}}} \frac{T_{o}^{3 / 2}}{D^{2}}\left[1+\sqrt{1+\frac{e V}{T_{o}}}\right]^{3} A
$$

where $\mathrm{D}$ is the gap spacing, $\mathrm{V}$ is the gap voltage, $\mathrm{T}_{\mathrm{o}}$ is the initial energy at which the electrons are injected into the gap ( $\sim$ gate voltage), e is the magnitude of the electron charge, $m_{e}$ is the electron rest mass, and $\varepsilon_{\mathrm{o}}$ is the free space permittivity. If $\mathrm{V}=0$ or $\mathrm{V} \ll \mathrm{T}_{\mathrm{o}}$ ("shorted anode") This equation indicates that there is a factor of 8 improvement over the classical 1-D Child-Langmuir current limit $\left(\mathrm{T}_{\mathrm{o}}=0\right)$.

Thus in the most simple one dimensional analysis, an EFES emitting $50 \mathrm{eV}$ electrons across a $1 \mathrm{~V} 1.5 \mathrm{~cm}$ gap (a typical sheath size for a neutral body in low earth orbit [Hastings, 1996]), the space charge current limit is $3 \mathrm{~mA} / \mathrm{cm}^{2}$. This is an effective lower limit to the ability of an FEA to emit charge (assuming that crossing the sheath is the primary limitation). So to get $1 \mathrm{~A}$ of emission would require a minimum of just over $300 \mathrm{~cm}^{2}$ of emitting area, i.e. a square emitter about $18 \mathrm{~cm}$ on a side. No accommodation need be made in this analysis for space charge limits increasing as the emitter area increases, because the 1-D analysis already assumes the worst case situation- an infinite sheet of charge.

Two and Three Dimensional Space Charge Limits

More detailed analysis indicates that significant improvement can be attained when you consider spreading of the emitted electrons in multiple dimensions. Luginsland [1996] suggests that the improvement by going to a long, thin emitter (modeled as an infinite strip, see figure below) would be a factor of 2 (Using a $0.5 \mathrm{~cm}$ wide emitter, $1.5 \mathrm{~cm}$ sheath). This was first determined using numerical analysis with XOOPIC and MAGIC, and later verified analytically [Lau, 2001]. This would reduce the required area to $150 \mathrm{~cm}^{2}$ for $1 \mathrm{amp}$ at $50 \mathrm{~V}$ emission.

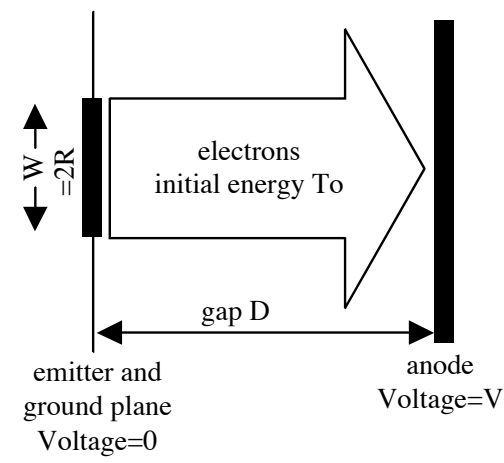

Figure 2. The scenario being analyzed for 2 dimensional space charge limits

$$
\frac{J_{c l}(2)}{J_{c l}(1)}=1+\frac{0.3145}{W / D}-\frac{0.0004}{(W / D)^{2}}
$$

Also at the University of Michigan, Lau [2001] extended his analytical agreement with the above 2D solution to the three dimensional case as given below:

$$
\begin{gathered}
\frac{J_{C L}(2)}{J_{C L}(1)} \cong 1+\frac{D}{4 R} \\
\frac{\text { and }}{J_{C L}(3)} \cong 1+\frac{D}{\pi W}+\left(\frac{1}{4}-\frac{1}{2 \pi}\right) \frac{D}{R}
\end{gathered}
$$

solved for situation where $\mathrm{R}>=\mathrm{W} / 2$, and $\mathrm{R} / \mathrm{D}>>1$. These solutions assume a gap size and/or magnetic field such that beam spreading is not a factor. They also assume that the charge density varies only with the emission direction, not laterally.

In the situation where $\mathrm{R} / \mathrm{D}<1$, as is likely to be the case for a space born EFES, another theoretical calculation by Humphries [1990] which does include beam spreading is given below (for a flat circular emitter with radius $\mathrm{r}_{\mathrm{b}}$ ). 


$$
\frac{J_{C L}(3)}{J_{C L}(1)}=\frac{\left[r_{b}^{2}+(D / 2)^{2}\right]}{r_{b}^{2}}=\left[1+\left(D / 2 r_{b}\right)^{2}\right]
$$

This equation suggests an improvement of 2.75 for a $1 \mathrm{~cm}^{2}\left(\mathrm{r}_{\mathrm{b}}=0.564 \mathrm{~cm}\right), 1.5 \mathrm{~cm}$ sheath case. Comparing back to our initial example this would dictate an EFES system with about $110 \mathrm{~cm}^{2}$ emission area, which is a marked improvement over the original 1D Child-Langmuir $300 \mathrm{~cm}^{2}$ area. The caveat is that this area must be in isolated $1 \mathrm{~cm}^{2}$ sections (spaced such that the gaps between emitters are large with respect to D) for the Humphries equation to apply.

The most advanced of the above equations consider only beam spreading from internal effects of the beam, as it moves away from a planar source to a planar collector in a DC fashion. Additional spreading occurs when external devices are in place, thus decreasing the density at the center where the virtual cathode first forms, and increasing the space charge limit. Further improvement can be gained when emission is spread over greater area with gaps between emitters. These are the sorts of modifications to the initial space charge limit calculations that will be considered below. These solutions exploit the fact that for this application the character of the beam is irrelevant as long as the electrons do not return to the spacecraft. For electric propulsion, or solar panel arc suppression, as long as the electrons move away into the surrounding plasma it does not matter if they are in a tight beam, which allows different solutions than the more commonly studied traveling wave tube amplifier, particle accelerator, plasma television, or other terrestrial applications.

\section{Particle-In-Cell Analysis and the XOOPIC Code}

Analytical solutions of more complicated geometries rapidly become intractable, whereas a wide variety of arbitrary situations can be simulated quickly and easily with PIC techniques. In a particle-in-cell simulation, a computer tracks the location and velocity of particles at arbitrary positions, and the electric and magnetic fields generated by those particles and the surrounding structures on a grid established in the simulation space. A cyclic iteration is done to update the velocity, position, and field values with every time step, as described by the following figures.

\begin{tabular}{|c|c|c|c|c|c|}
\hline & \multicolumn{3}{|c|}{ Exy, Bxy } & \multirow{2}{*}{$\underbrace{e^{V x y}}_{(x y)}$} & \\
\hline & $\mathrm{e}$ & $\mathrm{e}$ & $\mathrm{e}$ & & \\
\hline e & $\mathrm{e}$ & & $\mathrm{e}$ & $\mathrm{e}$ & $\mathrm{e}$ \\
\hline & e & $\mathrm{e}$ & e & $\mathrm{e}$ & $\mathrm{e}$ \\
\hline $\mathrm{e}$ & & & e & & $\mathrm{e}$ \\
\hline & & $\mathrm{e}$ & & & \\
\hline
\end{tabular}

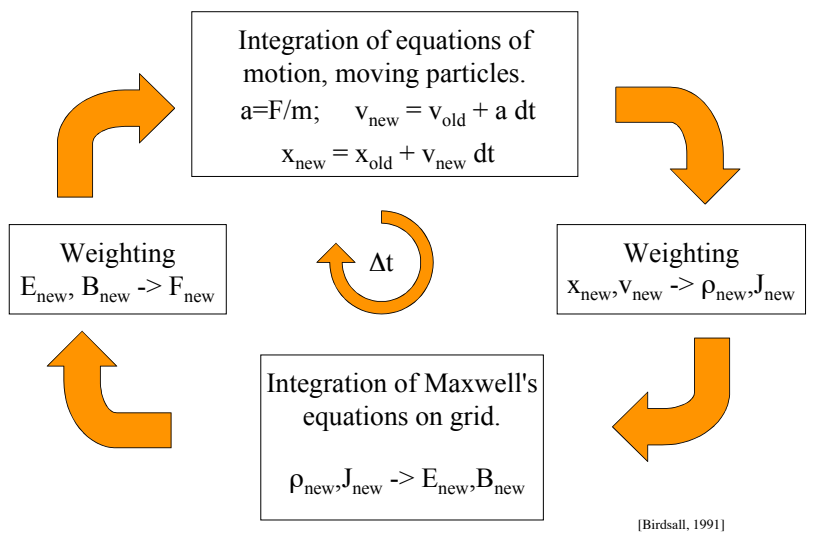

Figure 3. The cells and grids where parameters are tracked in PIC simulations, and the cycle used to iterate those parameters

With each cycle the computer sums all the charge within a few cells of an intersection to generate field values for that intersection. Then the fields at the four intersections are averaged or interpolated to be applied to each particle in the cell surrounded by the four intersections.

The larger the cells, the greater the error, as the fields are calculated further and further away from the particles effected. The larger the time step the more cells the particles pass through before the field effect is recalculated, further increasing the error. Additionally the number of particles used in the simulation- each simulated particle typically representing hundreds to hundreds of thousands of real particles- effects the accuracy of the simulation. On 
the other hand, having large cells, long time steps, and fewer particles accelerates the simulation allowing you to test larger parameter spaces and more geometries. These parameters are chosen as a balance between accuracy and simulation speed. Results used here were found to be self consistent with simulation parameter variations to within about $10 \%$, which should be sufficient to support the conclusions made.

\section{XOOPIC Particle-In-Cell Simulation Code}

The code used for results in this thesis is XOOPIC, developed out of Berkley by John Verboncoeur. [Verboncoeur, 1993] XOOPIC is a 2.5D code (i.e. tracking 2 physical dimensions and 3 velocity dimensions). XOOPIC runs on Unix, Linux, and other Unix based systems such as Macintosh OS X. There is a version called OOPIC for the PC as well. The code is widely used and well supported.

The following figure shows the setup for most of the XOOPIC simulations. The simulation uses radial symmetry along the axis of the emitted beam.

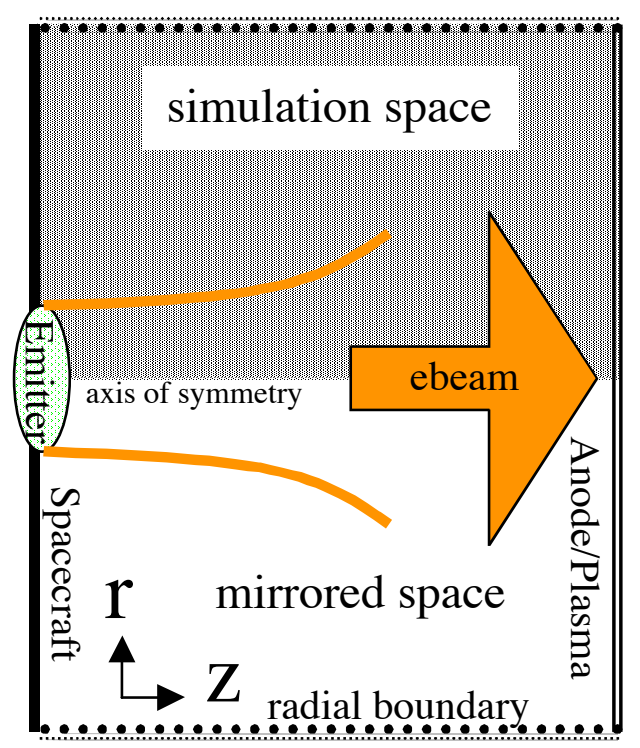

Figure 4. Setup of the simulation space in XOOPIC. The simulation is $2.5 \mathrm{D}, 2$ dimensions in space, 3 dimensions in velocity.

Simulations in XOOPIC were compared to results obtained with a thermionic emitter in a vacuum chamber. In the experimental setup the electrons were accelerated away from the emitter with a single grid a few millimeters from that emitter. The grid was grounded, while the emitter was biased negative to 210 volts. The electrons were collected at an anode 10 centimeters away. The following plot shows a comparison between simulation and experiment for a constant emission voltage and a varying anode voltage. The agreement is reasonable. There is a slope in the experiment above the flattening in the simulation data because the simulation uses a constant $29.5 \mathrm{~mA}$ beam, while the current in the experiment is determined not only by the space charge limit of the fixed bias between the acceleration grid and the thermionic emitter, but also by the electric field between the anode and the grid which leaks through the grid to the space between the emitter and the grid. So as the anode bias is changed, the beam current also changes, especially at large anode biases. The jump around $-105 \mathrm{~V}$ is due to a ranging switch of a voltage sensor on the anode which changes the resistance of an alternate current path. Additional sources of discrepancy are the varying physics of current collection at the acceleration grid, variable emission from the emitter due to chamber contamination, and misalignment of the emitter. 


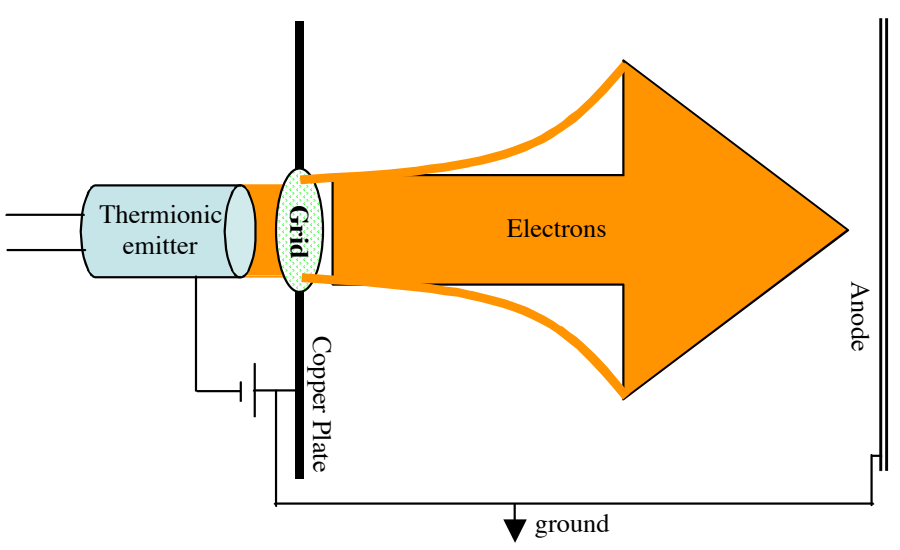

Figure 5. Schematic diagram of the experimental setup

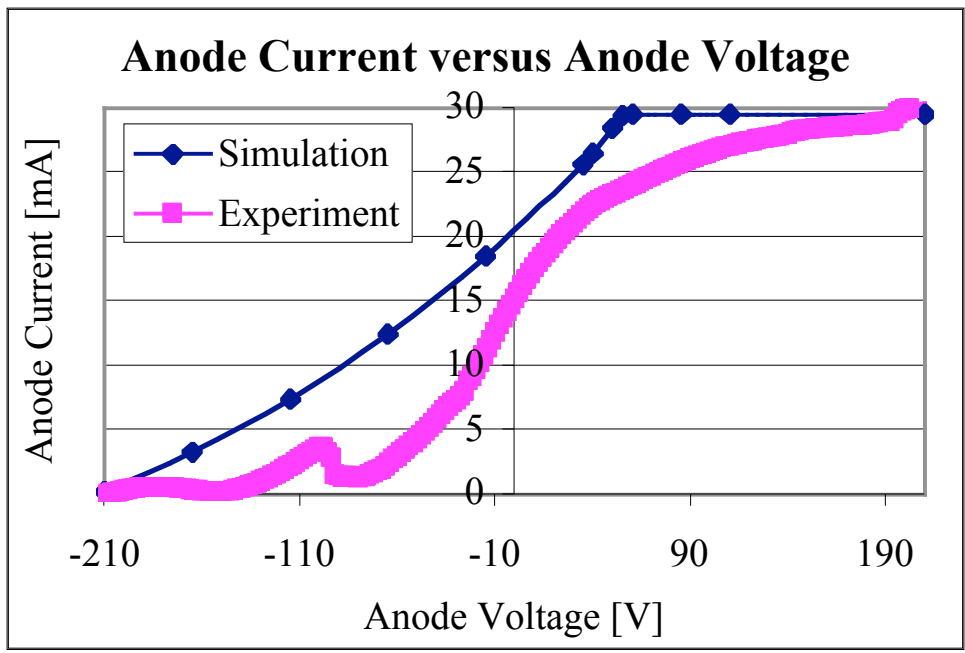

Figure 6. comparison of emission in a vacuum chamber to experimental results

\section{Effects of Emitter Size}

The following plot shows variation in the space charge limit not as the velocity is varied, but as the emitter width is varied. The space charge limit in $\mathrm{mA}$ is shown for reference but the key point is the factor of improvement over the 1D situation (right side scale). Lau's analysis agrees with the XOOPIC analysis (and Humphries) for wider emitters, but as the emitter size drops down towards the very small, Humphries analysis is a much better predictorwhich is what in his paper Lau says would be the case, as his solution was only done for the case where the emitter was wide with respect to the gap size. 


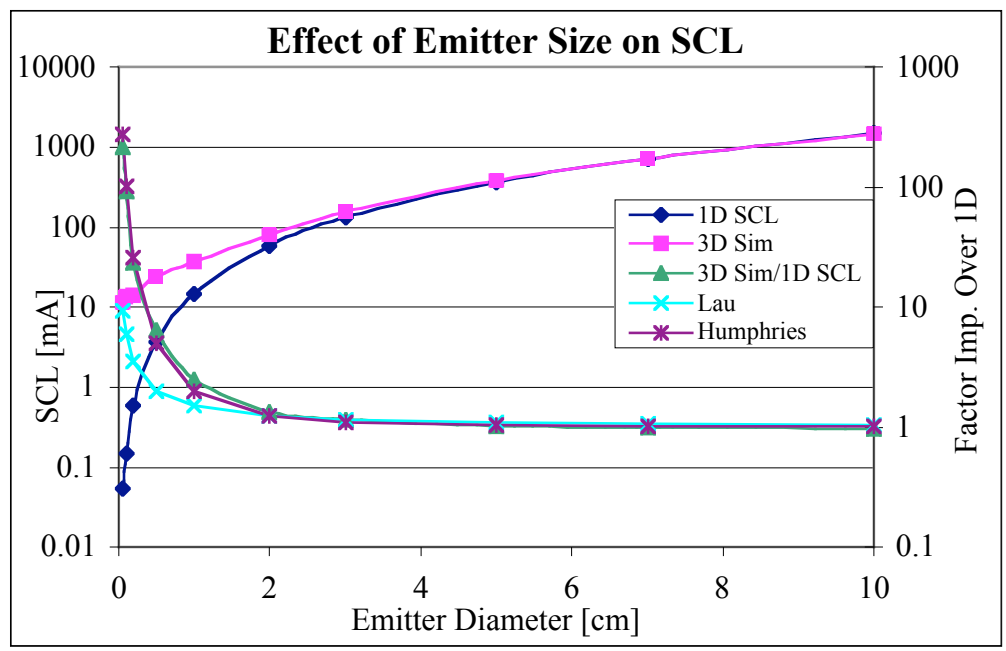

Figure 7. Plot of the space charge limit as a function of emitter size.

The agreement with Humphries analysis is also taken as support that the XOOPIC simulations are sufficiently accurate to make determinations about the space charge limit in various situations. In most of the analysis done the results will be presented as factors of improvement over the 1D space charge limit, or the 3D space charge limit without the improvement technique. An absolute shift of the results up or down is not taken to be as significant as a change in the shape of the solution as the parameter of interest is varied.

The important conclusion of these results is that beam spreading from small emitters allows a vast improvement in the space charge limit over wide emitters. For a spacecraft where emission power is the most critical feature, the best solution may be a number of button sized emitters spread around the spacecraft. The space between these emitters must be sufficiently large that their respective beams do not overlap enough for the combined density to exceed the SCL, as discussed next.

\section{Emitter Spacing}

A critical question in the design of an EFES is how close emitters can be placed to one another. It has already been shown that small emitters operating in isolation with a large amount of room for the beam to spread benefit from a greatly improved SCL per unit emitter area relative to much larger emitters. By extension, the best case scenario would be a single tip of a Spindt type micro-fabricated emitter. In isolation it would not emit much, but the efficiency per unit surface area of that one tip would be immense. In contrast, the worst case scenario is a large planar emitter (in this case "large" need only be a few centimeters wide) where the SCL is almost identically the planar 1D Child-Langmuir solution.

Thus if the only objective were to maximize emitted current per emitter area, the ideal solution would be many very small emitters widely spread. What is less obvious is the tradeoff from a systems designer point of view of whether to use one large emitter or many smaller ones or where to choose the balance in between. An EFES designer must build a system that emits the required amount of current within the physical constraints of the systempower, mass, volume, and possibly most significantly, spacecraft surface area. Clearly smaller emitters are more efficient, but how far apart must they be to gain this efficiency? 


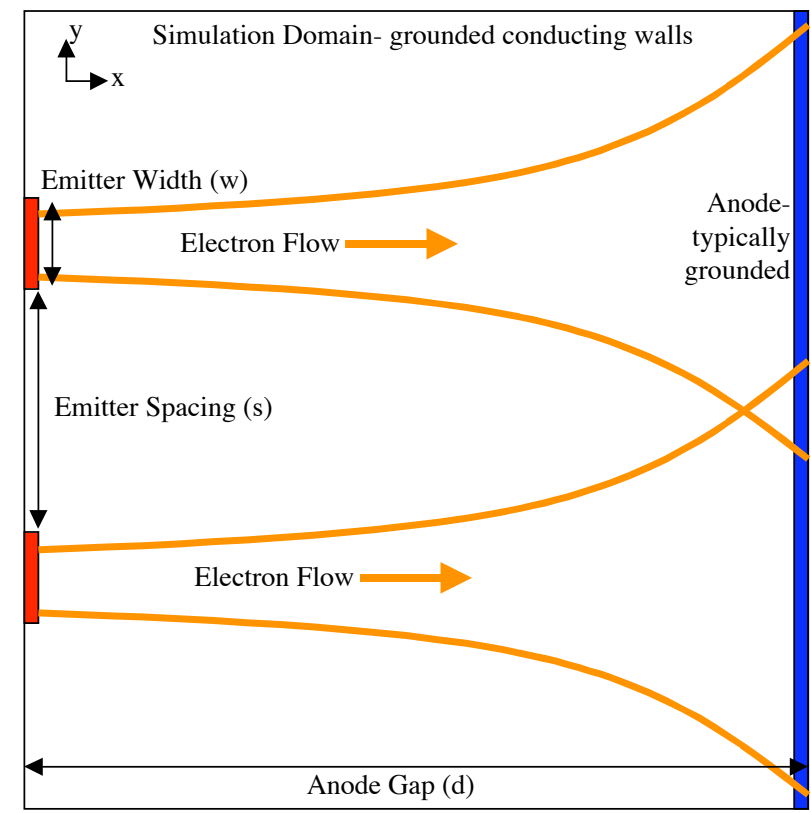

Figure 8. 2 Dimensional $\mathrm{x}-\mathrm{y}$ simulation setup for analysis of spacing effects in XOOPIC. Two emitters are placed on the left wall of the simulation domain a distance s apart. They emit electrons with an initial velocity in the $\mathrm{x}$ direction to the right wall. For simplicity, all walls, including the anode, are held at ground.

The following plots shows the effect of proximity on two emitters, as calculated using XOOPIC in 2D mode. Current is emitted in the $\mathrm{x}$ direction. The emitters are simulated as infinitely tall (for purposes of current calculation, they are $1 \mathrm{~m}$ tall) and only spreading in the y dimension is considered. The widths and spacings between emitters are shown in $\mathrm{cm}$. It is apparent that the effect of the spacing between emitters levels off at some point, a point dependant both on the width of the emitter (w) and the emitter-anode gap (d), but not on the emission velocity. The default anode gape is $2 \mathrm{~cm}$. The width of the simulation domain is the size of the emitters plus the gap between plus five centimeters on the outside of each emitter.

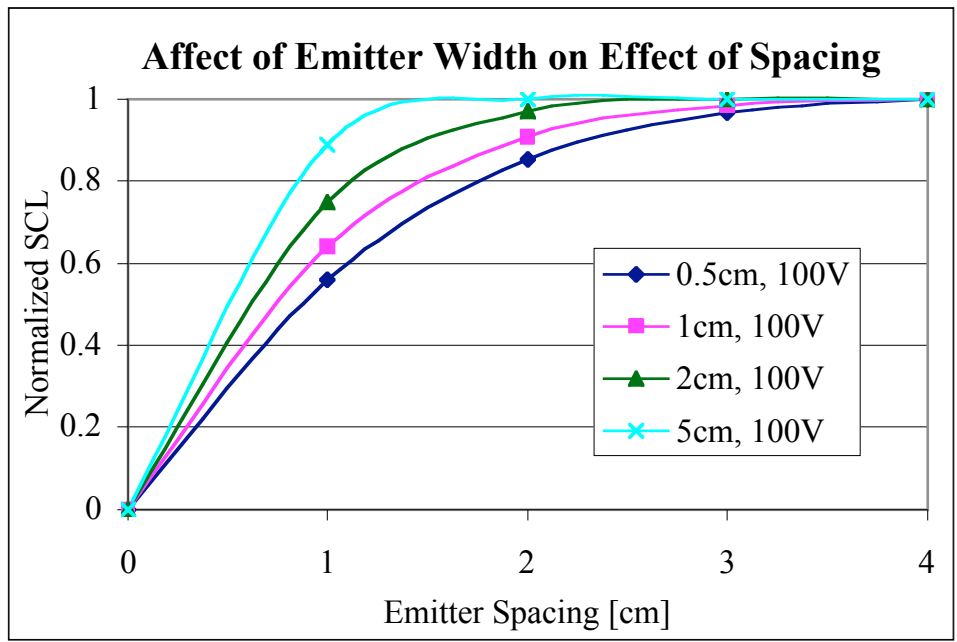

Figure 9. This figure shows the space charge limit as determined by XOOPIC. The data is normalized separately for each emitter size (each line) such that zero is the limit when the emitters are adjacent, and 1 is the maximum SCL attained at wide separation (s). Emission is at 100V. Gap (d) is $2 \mathrm{~cm}$. Observe that the wider the emitter, the sooner additional spacing becomes irrelevant. 


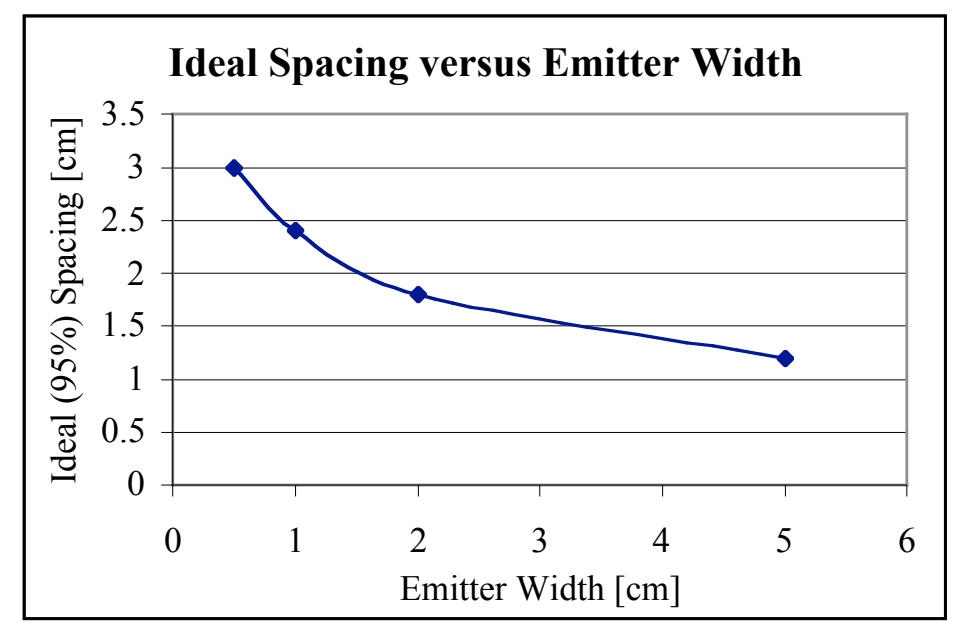

Figure 10. Condensed from the plot above, this shows the trend in ideal spacing for a pair of emitters (the point at which the emitter SCL is $95 \%$ of what it would be for each emitter in isolation) versus emitter width.

\section{The Effect of Defocus Rings}

Adding a biased metal cone around a planar circular emitter increases the amount of current that can be emitted at a given emission velocity before the space charge limit is reached. Even with a grounded ring (zero volt bias), the space charge limit is improved. The general explanation for this improvement is that a proximate cylindrical conductor increases the strength of the radial electric field and thus the radial spreading force is larger and the beam spreads faster. Beam spreading reduces the density in the center of the beam where the virtual cathode forms. The lower the density the more current it takes for the virtual cathode to form, and thus for the space charge limit to take effect. One can think of the defocus ring as a more proximate reference point than infinity for the electric field lines, or one can think of the positive charge forming on the interior of the ring (mirroring the negative charge of the emitted beam) thus increasing the field strength as in a capacitor. Regardless the beam is spread and the space charge limit is improved with no power dissipation by the ring (though the overall system cost can be nonzero depending on the cost of biasing the spacecraft relative to the surrounding plasma).

The following diagram shows the simulation setup with the defocus ring in place. The next figures show screenshots of the simulation in progress.

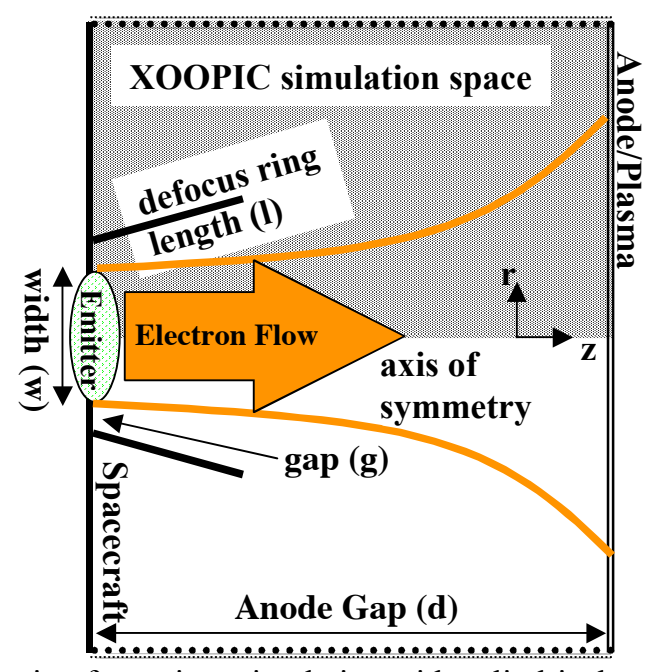

Figure 11. Configuration for emitter simulation with cylindrical symmetry in XOOPIC.

The following figures illustrate quite clearly the benefit of a defocus ring. The simulation is of $30 \mathrm{~mA}$ of current being emitted from a $1 \mathrm{~cm}^{2}$ emitter across a $2 \mathrm{~cm}$ gap at $100 \mathrm{~V}$. The defocus ring is $0.5 \mathrm{~cm}$ long, $0.1 \mathrm{~cm}$ away from the 
emitter, and at a $15^{\circ}$ angle off of parallel to the emission beam. The ring is biased at zero volts as are all of the surrounding walls. Without the defocus ring (left figure) you get space charge limited flow within $15 \mathrm{~ns}$. With the defocus ring the beam is clearly below the space charge limit even after $150 \mathrm{~ns}$.
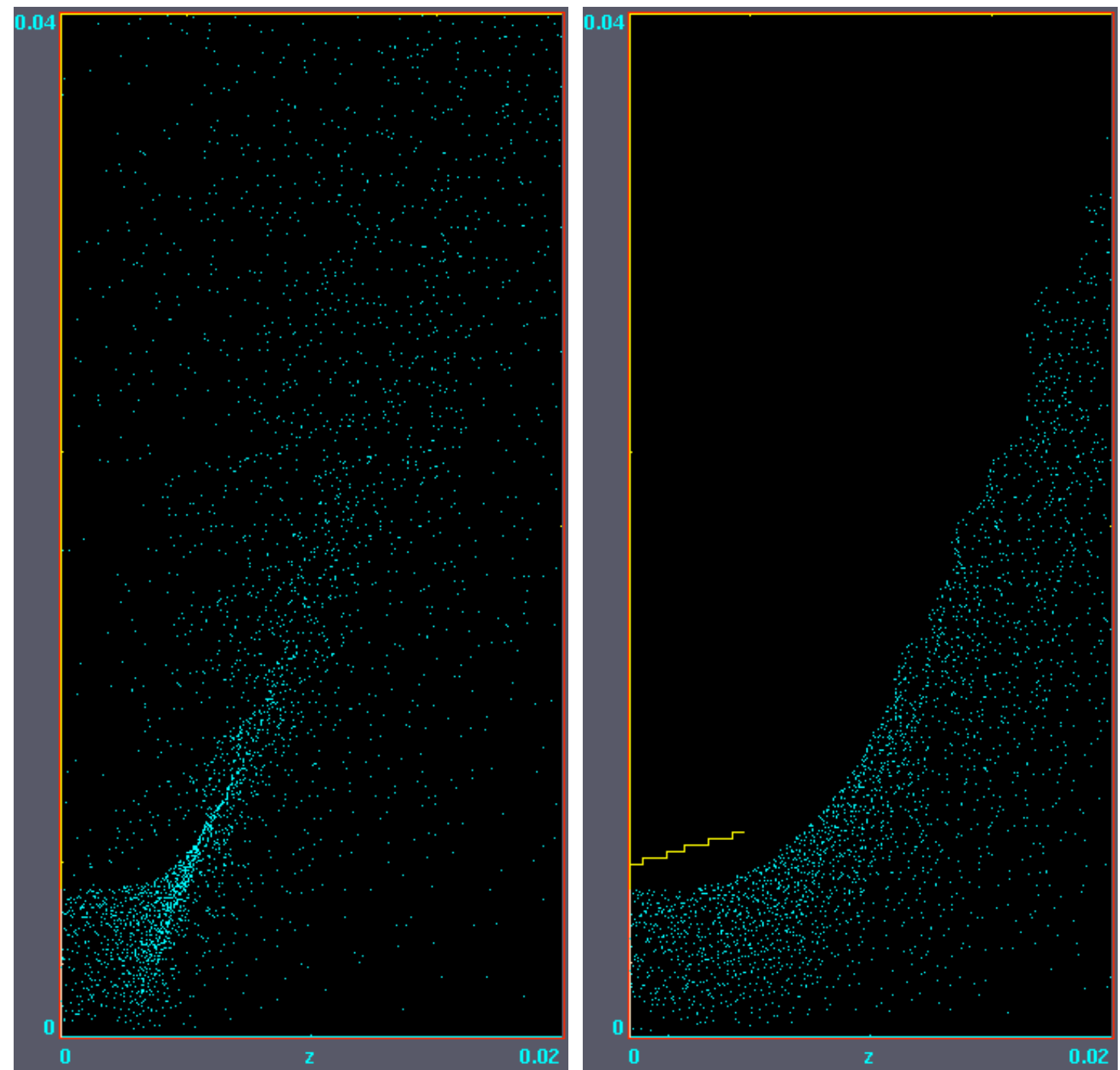

Figure 12. Improvement to emission characteristics via the addition of a grounded ring around the emitter.

The voltage on the ring affects the benefit, with higher voltages conferring greater improvement to the space charge limit. Negative voltages worsen the space charge limit- and in fact this configuration is similar to the beam optics used in electron guns where the objective is confine and regulate the electron beam in a uniform column [Reiser, 1994], exactly opposite to the objective of avoiding space charge limits. One specific example of such is a Pierce Cathode- where a ring surrounding the emitter at an angle of $67^{\circ}$ is used to create a perfectly collimated beam.

There is a strong dependence upon the angle of the defocus ring in determining it's effectiveness at alleviating space charge limits. The following plot shows the results of simulations with the above parameters $(100 \mathrm{~V}$ emission across a $2 \mathrm{~cm}$ gap) with defocus rings biased from -100 to $+100 \mathrm{~V}$ at varying angles around the emitter. The rings are all $0.1 \mathrm{~cm}$ away from the emitter and $0.5 \mathrm{~cm}$ long. The results clearly show that the closer to the emitted beam the rings are (smaller angles), the more effective. The limit occurs when the ring is close enough, or the beam spread far enough, that the beam contacts the ring and the ring collects charge; at this point it is no longer effectively assisting in moving charge away from the spacecraft. A best case improvement of $160 \%$ (factor of 2.5 ) is shown between no 
ring and a $15^{\circ} 100 \mathrm{~V}$ ring. Even with no bias on the ring (a simpler cheaper system), an improvement of $40 \%$ (factor of 1.4) can be obtained with a grounded ring.

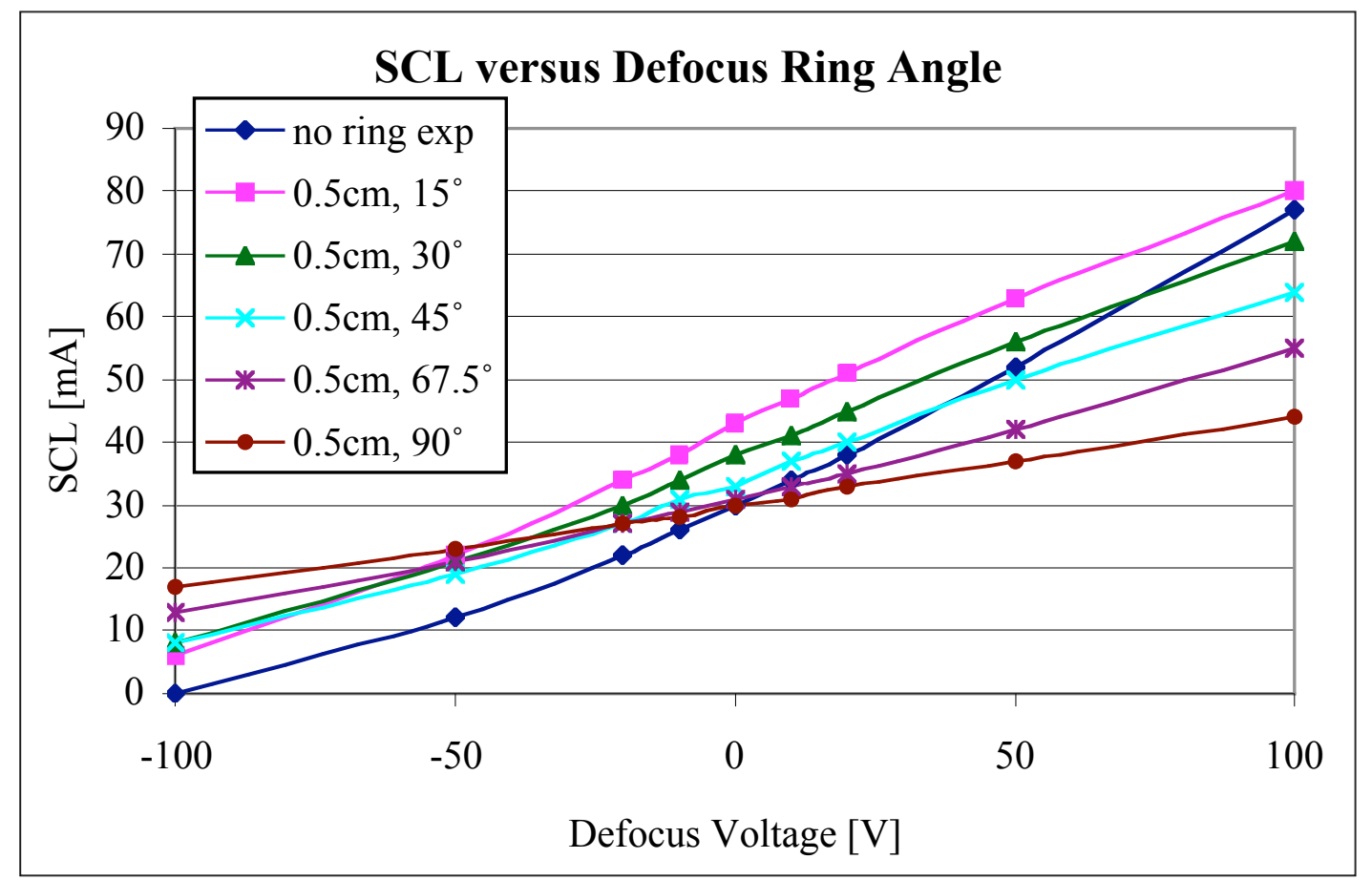

Figure 13. a comparison of the effect of a defocus ring on the SCL, versus the voltage on the ring, for various ring angles. The defocus length is $0.5 \mathrm{~cm}$, and the ring starts $0.1 \mathrm{~cm}$ away from the emitter.

The following plot shows the effect of gap size and defocus length on the effectiveness of the defocus ring.
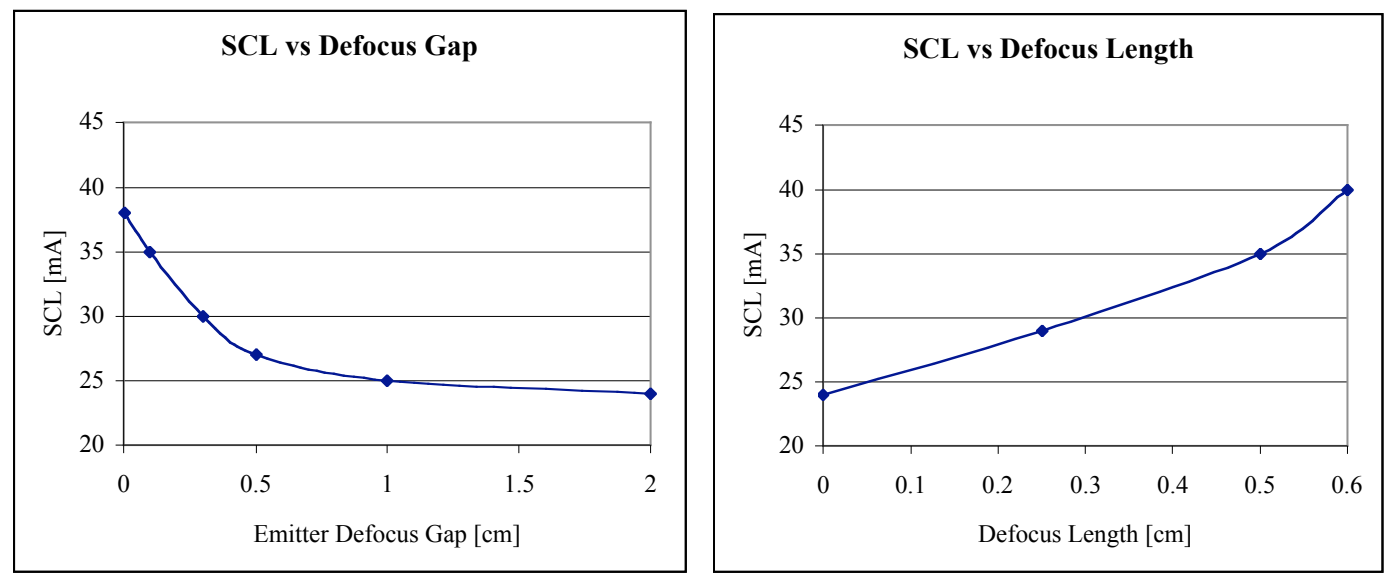

Figure 14. Effect on the space charge limit of variation of the defocus gap and defocus length. Simulations are done with a $1 \mathrm{~cm}^{\wedge} 2$ circular emitter emitting at $100 \mathrm{~V}$ across a $2 \mathrm{~cm} 0 \mathrm{~V}$ gap.

The effect of the gap size and the defocus length is similar- the closer the beam gets to the defocus, the stronger it's effect. This occurs both in the case where the defocus is positioned closer to the emitter and the case where the defocus continues out to the point where the beam has spread almost to within contact of it. In either case, the gap and length are limited from exponential increases at the zero point by contact between the beam and the defocus. All analysis are extended only to this point, as it obfuscates the results to interpret a scenario where only part of the current is escaping. The defocus length plot above ends at $0.6 \mathrm{~cm}$ because for these parameters beyond that length the beam is partially collected by the defocus. 
The effectiveness of a defocus ring varies inversely with the size of the emitter- very large emitters receive little benefit, adding another tradeoff to the system level decisions about the sizing and spacing of emitters.

\section{Conclusion}

For emission scenarios where the beam power/velocity is low relative to the beam current, the size and shape and surrounding geometry of the emitter can have a significant impact on the efficiency of the emitter. Scenarios such as these occur, for example, on small spacecraft with small thruster systems using cold cathode field emitters. For small spacecraft power is inherently limited so the efficiency of the emitter is all the more important. In these and similar situations designing the emission system with the techniques presented here to mitigate space charge limits could provide essential power savings.

\section{Acknowledgments}

This work is being funded by a NASA-MSFC GSRP student grant.

\section{References}

Child, C. D., "Discharge from Hot CaO", Phys. Rev., ser. 1, vol. 32, 1911

K. L. Jensen, Physics of Plasmas 6, 2241, (1999).

Gilchrist B. K., Jensen, A. Gallimore, J. Sorensa, Field-Emitter Array Cathodes (FEACs) for Space-Based Applications: An Enabling Technology, white paper, The University of Michigan and The Naval Research Laboratory, 1999

Langmuir, I. Phys. Rev. Ser. II, 2, 450 (1913)

Lau, Y. Y., "A simple Theory on the Two-Dimensional Child-Langmuir Law", Phys. Rev. Lett. 87, 278301, 2001.

Lieberman, Lichtenberg, Principles of Plasma Discharges and Materials Processing, John Wiley \& Sons, Inc., 1994

Luginsland, J., S. McGee, and Y. Y. Lau, IEEE Trans. Plasma Sci., 26, p.p. 901-904, 1998.

Luginsland, J. W., Y. Y. Lau, R. M. Gilgenbach, Two-dimensional Child-Langmuir law, Phys. Rev. Lett., 77, p. $4668,1996$.

Marrese, C., Compatibility of field emission cathode and electric propulsion technologies: theoretical and experimental performance evaluations and cathode requirements, Ph.D., University of Michigan, 1999.

NRL Plasma Formulary published by the Naval Research Laboratory, 1998 edition. NRL/PU/6790--98-358

Verboncoeur, J. P., M. V. Alzes, V. Vahedi, C. Birdsall, Simultaneous Potential and Circuit Solution for 1-D Bounded Plasma Particle Simulator Codes, J. Comp. Phys., V. 104, p321-328, 1993

Winglee, R.M., P.L. Pritchett, "Space Charge Effects During the Injection of Dense Electron Beams Into Space Plasmas", Journal of Geophysical Research, Vol. 92, No. A6, Pages 6114-6126, 1987 